\title{
Structures of Grixazone A and B, A-Factor-dependent Yellow Pigments Produced under Phosphate Depletion by Streptomyces griseus
}

\author{
Yasuo Ohnishi, Yasuhide Furusho, Tatsuichiro Higashi, Hyo-Kon Chun ${ }^{\dagger}$, Kazuo Furihata ${ }^{a}$, \\ ShOHEI SAKUdA ${ }^{\mathrm{a}}$ and Sueharu HorinOUChI* \\ Department of Biotechnology and a Department of Applied Biological Chemistry, \\ Graduate School of Agriculture and Life Sciences, The University of Tokyo, \\ Bunkyo-ku, Tokyo 113-8657, Japan \\ (Received for publication December 11, 2003)
}

\begin{abstract}
A-factor (2-isocapryloyl-3R-hydroxymethyl- $\gamma$-butyrolactone) acts as a microbial hormone that induces morphological development and secondary metabolism in Streptomyces griseus. A diffusible yellow pigment is produced by $S$. griseus in an A-factor-dependent manner under phosphate depletion. Detailed analysis of the pigment production by S. griseus cultivated in minimal liquid medium containing different concentrations of phosphate showed that the pigment was actively produced in the presence of low concentrations of phosphate and the production of the pigment was completely repressed in the presence of $2.5 \mathrm{mM} \mathrm{KH}_{2} \mathrm{PO}_{4}$. $\mathrm{HPLC}$ analysis of the culture supernatant showed that the pigment consisted of two major, structurally related compounds and they were produced at different ratios depending on the concentration of phosphate in the medium. The structures of the two major compounds, designated as grixazone $\mathrm{A}$ and $\mathrm{B}$, were determined by spectroscopic analyses as 1-[[2-(acetylamino)2-carboxyethyl]thio]-2-amino-3-oxo-8-formyl-3H-phenoxiazine and 1-[[2-(acetylamino)-2carboxyethyl]thio]-2-amino-3-oxo-8-carboxyl-3H-phenoxiazine, respectively. Grixazone A was a novel compound, although grixazone B was reported in a patent as a parasiticide produced by Streptomyces sp. DSM3813.
\end{abstract}

Many Streptomyces species produce $\gamma$-butyrolactones, most of which have been shown to act as signals for the onset of antibiotic production. A-factor (2-isocapryloyl-3Rhydroxymethyl- $\gamma$-butyrolactone) is the most intensively and extensively studied $\gamma$-butyrolactone and is required for not only antibiotic production but also morphological differentiation in $S$. griseus $^{1,2)}$. A-factor is produced in a growth-dependent manner and accumulated until the end of exponential growth. It triggers the A-factor regulatory cascade by inducing the transcription of $a d p A$, which encodes a transcriptional activator. Induction of $a d p A$ requires binding of $\mathrm{A}$-factor to the A-factor-receptor ArpA, which has bound and repressed the promoter of $a d p A$. Binding of A-factor dissociates ArpA from the promoter ${ }^{3)}$. Although AdpA was originally identified as an A-factordependent transcriptional activator for $s t r R$ encoding the pathway-specific transcriptional activator for streptomycin biosynthesis, AdpA has been shown to induce the transcription of a number of genes involved in secondary metabolism and morphogenesis ${ }^{4 \sim 8)}$, thus forming an AdpA regulon.

We have observed that $S$. griseus produces a diffusible yellow pigment when cultured on a phosphate-depleted agar plate. Because neither an A-factor-deficient strain (HH1) nor an $a d p A$-disrupted strain produces the yellow pigment ${ }^{4)}$, expression of the biosynthetic genes for the yellow pigment is probably under the control of A-factor and AdpA. In addition, detailed analysis of the production of the pigment by $S$. griseus cultivated in minimal liquid medium containing different concentrations of phosphate revealed that the pigment is produced only in medium containing a low concentration of phosphate. This means that the production of the yellow pigment is switched-on by A-factor and controlled by the phosphate concentration of

* Corresponding author: asuhori@mail.ecc.u-tokyo.ac.jp

${ }^{\dagger}$ Present address: Korea Research Institute of Bioscience and Biotechnology, Yusong, Taejon 305-600, Korea. 
the medium.

Because of the regulation of the pigment production by both A-factor and phosphate, the biosynthetic gene cluster for the pigment is a useful material for elucidating the molecular mechanisms by which $\gamma$-butyrolactones and phosphate control secondary metabolite formation. As a first step for these studies, we have determined the chemical structure of the pigment, which actually turns out to consist of two major compounds. In this paper, we describe detailed analysis of the pigment production in response to phosphate and the chemical structures of the two major compounds in the yellow pigment.

\section{Materials and Methods}

\section{Strain and Culture Conditions}

S. griseus IFO13350 was obtained from the Institute of Fermentation, Osaka, Japan (IFO). S. griseus was grown at 26.5 or $30^{\circ} \mathrm{C}$ in YMPD medium (yeast extract [Difco], $0.2 \%$; meat extract [Kyokuto], $0.2 \%$; Bacto-peptone [Difco], $0.4 \% ; \mathrm{NaCl}, 0.5 \% ; \mathrm{MgSO}_{4} \cdot 7 \mathrm{H}_{2} \mathrm{O}, 0.2 \%$; and glucose, $1 \%$; pH 7.2) and SMM medium (glucose, $0.9 \%$; L-asparagine, $0.9 \%$; $\left(\mathrm{NH}_{4}\right)_{2} \mathrm{SO}_{4}, \quad 0.2 \%$; Trizma base, $0.24 \% ; \mathrm{NaCl}, 0.1 \% ; \mathrm{K}_{2} \mathrm{SO}_{4}, 0.05 \% ; \mathrm{MgSO}_{4} \cdot 7 \mathrm{H}_{2} \mathrm{O}, 0.02 \%$; $\mathrm{CaCl}_{2}, 0.01 \%$; and trace element solution ${ }^{9)}, 1 \%, \mathrm{pH}$ 7.2) containing different concentrations $(0 \sim 2.5 \mathrm{mM})$ of $\mathrm{KH}_{2} \mathrm{PO}_{4}$.

Yellow Pigment Production in Liquid Medium Containing Different Concentrations of Phosphate

S. griseus IFO13350 cultivated on YMPD agar was inoculated into $100 \mathrm{ml}$ of YMPD in a $500 \mathrm{ml}$ shaking-flask and precultured at $30^{\circ} \mathrm{C}$ for 2 days with reciprocal shaking. The mycelium was washed three times with SMM and homogenized by a glass homogenizer. Then $1 / 100$ volume of the mycelium was inoculated into $100 \mathrm{ml}$ of SMM containing different concentrations $(0 \sim 2.5 \mathrm{mM})$ of $\mathrm{KH}_{2} \mathrm{PO}_{4}$ in a $500-\mathrm{ml}$ shaking-flask and cultured at $26.5^{\circ} \mathrm{C}$ for 5 days with reciprocal shaking. A portion $(5 \mathrm{ml})$ of the culture was taken everyday and the wet weight of the mycelium and the absorbance at $433 \mathrm{~nm}\left(\mathrm{~A}_{433}\right)$ of the culture supernatant were measured.

\section{Analysis of the Yellow Pigment by HPLC}

Mycelium of S. griseus precultured in $100 \mathrm{ml}$ of YMPD as described above was washed three times with SMM and homogenized. Then $1 / 10$ volume of the mycelium was inoculated into 1 liter of SMM containing different concentrations $(0.25 \sim 1 \mathrm{~mm})$ of $\mathrm{KH}_{2} \mathrm{PO}_{4}$ in a 5 liter-flask with baffles and cultured at $26.5^{\circ} \mathrm{C}$ for 5 days with rotary shaking. The culture supernatant was filtered through a $0.22 \mu \mathrm{m}$ membrane and a portion $(10 \mu \mathrm{l})$ was applied to HPLC equipped with a reverse-phase column (Senshu Pak PEGASIL-B ODS, $4.6 \times 250 \mathrm{~mm}$, Senshu Kagaku) and a photodiode array detector (model 996, Waters). It was developed at a flow rate of $1 \mathrm{ml} /$ minute with a linear gradient from solvent $\mathrm{A}\left(\mathrm{H}_{2} \mathrm{O}-\mathrm{CH}_{3} \mathrm{CN}, 9: 1\right)$ to solvent $\mathrm{B}$ $\left(\mathrm{CH}_{3} \mathrm{CN}\right)$ for 20 minutes. Both solvent $\mathrm{A}$ and $\mathrm{B}$ contained $0.1 \%$ trifluoroacetic acid (TFA).

\section{Isolation of Grixazone A}

Mycelium of S. griseus precultured in $100 \mathrm{ml}$ of YMPD as described above was collected by centrifugation and homogenized. Then 1/10 volume of the mycelium, which included a portion of YMPD medium, inoculated into 1 liter of SMM without $\mathrm{KH}_{2} \mathrm{PO}_{4}$ in a 5 liter-flask with baffles and cultured at $26.5^{\circ} \mathrm{C}$ for 3 days with rotary shaking. The culture supernatant was filtered through an $8.0 \mu \mathrm{m}$ membrane. Grixazone A in the filtrate (total, 12 liters) was absorbed on Diaion HP-20 resins in a column. After the column had been washed with $30 \%$ methanol, grixazone A was eluted with $70 \%$ methanol. The eluate was concentrated in vacuo to dryness, and the residue was dissolved in $10 \mathrm{ml}$ of methanol. The solution was then applied on a gel filtration column (Sephadex LH-20, Amersham Pharmacia) equilibrated with methanol. The fractions with a red-yellow color were collected and concentrated in vacuo to dryness. This crude material was purified by two steps of preparative HPLC (1st step: column: Senshu Pak ODS-5251-SH, 20 $\times 250 \mathrm{~mm}$, Senshu Kagaku; mobile phase: gradient elution of $10 \sim 100 \%$ methanol in 30 minutes; flow rate: $4 \mathrm{ml} /$ minute, 2 nd step: column: Capcell Pak $\mathrm{C}_{18}, 10 \times 250 \mathrm{~mm}$, Shiseido; mobile phase: gradient elution of $10 \sim 100 \% \mathrm{CH}_{3} \mathrm{CN}$ in $0.1 \%$ TFA in 20 minutes; flow rate: $4 \mathrm{ml} /$ minute) to afford $4.7 \mathrm{mg}$ of grixazone A (1).

1: FABMS (glycerol matrix) $m / z 402(\mathrm{M}+\mathrm{H})^{+}$; HRESIMS $m / z 402.0772(\mathrm{M}+\mathrm{H})^{+}$(Calcd for $\mathrm{C}_{18} \mathrm{H}_{16} \mathrm{~N}_{3} \mathrm{O}_{6} \mathrm{~S}$, 402.0760); UV $\lambda_{\max } \mathrm{nm}(\varepsilon)$, (DMSO): $270(17,100), 414$ (14,800), 436 (15,200); ${ }^{1} \mathrm{H}$ and ${ }^{13} \mathrm{C}$ NMR (Table 1).

\section{Isolation of Grixazone B}

Mycelium of S. griseus precultured in $100 \mathrm{ml}$ of YMPD as described above was washed three times with SMM and homogenized. Then $1 / 10$ volume of the mycelium was inoculated into 1 liter of SMM containing $0.75 \mathrm{mM} \mathrm{KH}_{2} \mathrm{PO}_{4}$ in a 5 liter-flask with baffles and cultured at $26.5^{\circ} \mathrm{C}$ for $5 \sim 7$ days with rotary shaking. The culture supernatant was filtered through an $8.0 \mu \mathrm{m}$ membrane. Grixazone B in the 
Table 1. NMR Assignments of $\mathbf{1}$ and 2. ${ }^{\mathrm{a}}$

\begin{tabular}{|c|c|c|c|c|c|c|}
\hline \multirow[b]{2}{*}{ C-No. } & \multicolumn{3}{|c|}{1} & \multicolumn{3}{|c|}{2} \\
\hline & $\delta_{\mathrm{C}}$ & $\delta_{\mathrm{H}}$ & HMBC & $\delta_{\mathrm{C}}$ & $\delta_{\mathrm{H}}$ & HMBC \\
\hline 1 & 99.3 & & & 98.4 & & \\
\hline 2 & $150.3^{\mathrm{b}}$ & & & $150.1^{\mathrm{e}}$ & & \\
\hline $2-\mathrm{NH}_{2}$ & & 7.25 br.s & & & $7.20 \mathrm{br}$. & \\
\hline 3 & 178.7 & & & 178.9 & & \\
\hline 4 & 104.4 & $6.47 \mathrm{~s}$ & Observed $^{d}$ & 104.1 & $6.45 \mathrm{~s}$ & Observed $^{d}$ \\
\hline $4 a$ & $147.8^{\mathrm{b}}$ & & & $147.6^{e}$ & & \\
\hline $5 a$ & 145.9 & & & 144.8 & & \\
\hline 6 & 116.9 & $7.68 \mathrm{~d}(8.5)$ & C-8 and/or 9a & 116.1 & $7.60 \mathrm{~d}(8.5)$ & $C-5 a, 8,9 a$ \\
\hline 7 & 128.6 & $7.79 \mathrm{dd}(8.5,2.0)$ & C-5a, 9, 11 & 129.7 & $8.00 \mathrm{dd}(8.5,2.0)$ & $C-5 a, 9,11$ \\
\hline 8 & $133.3^{\mathrm{c}}$ & & & 128.0 & & \\
\hline 9 & 130.8 & $8.31 \mathrm{~d}(2.0)$ & C-5a, 7, 11 & 129.5 & $8.26 \mathrm{~d}(2.0)$ & $C-5 a, 7,11$ \\
\hline $9 a$ & $133.5^{c}$ & & & 133.0 & & \\
\hline $10 \mathrm{a}$ & $150.3^{\mathrm{b}}$ & & & $150.1^{\mathrm{e}}$ & & \\
\hline 11 & 191.8 & $10.08 \mathrm{~s}$ & C-7, 8,9 & 166.3 & & \\
\hline 12 & 35.3 & $3.16 \mathrm{~d}(6.5)$ & C- 1,13 & 35.1 & $3.16 \mathrm{~d}(6.0)$ & $C-1,13,14$ \\
\hline 13 & 52.7 & $4.34 \mathrm{~m}$ & C- $12,14,15$ & 52.5 & $4.34 \mathrm{~m}$ & C- $12,14,15$ \\
\hline $13-\mathrm{NH}$ & & $8.17 \mathrm{~d}(6.0)$ & & & $8.19 \mathrm{~d}(7.0)$ & \\
\hline 14 & 172.3 & & & 172.1 & & \\
\hline Ac $(C=O)$ & 169.2 & & & 169.1 & & \\
\hline $\mathrm{Ac}\left(\mathrm{CH}_{3}\right)$ & 22.5 & $1.79 \mathrm{~s}$ & C- 15 & 22.4 & $1.79 \mathrm{~s}$ & C-15 \\
\hline
\end{tabular}

${ }^{\text {a }}$ Spectra were obtained in DMSO- $d_{6}$ on a JEOL GX-500. ${ }^{\mathrm{b}, c, e}$ May be exchanged.

${ }^{d} \mathrm{C}-2$ and $4 \mathrm{a}, \mathrm{C}-2$ and 10, C-4a and C-10, or C-2, 4a and 10.

Coupling constants in Hertz are given in parentheses.

filtrate (total, 6 liters) was absorbed on Diaion HP-20 resins in a column and eluted with water. The eluate was applied on a $\mathrm{C}_{18}$ cartridge column (Sep-Pak Vac35cc). After the cartridge had been washed with water, grixazone $\mathrm{B}$ was eluted with $20 \% \mathrm{CH}_{3} \mathrm{CN}$ from the cartridge. The eluate was concentrated in vacuo and lyophilized. This crude material was purified by two steps of preparative HPLC (1st step: column: Senshu Pak ODS-5251-SH, 20×250 mm, Senshu Kagaku; mobile phase: gradient elution of $10 \sim 100 \%$ $\mathrm{CH}_{3} \mathrm{CN}$ in $0.1 \%$ TFA in 30 minutes; flow rate: $4 \mathrm{ml} /$ minute, 2nd step: column: Capcell Pak $\mathrm{C}_{18}, 10 \times 250 \mathrm{~mm}$, Shiseido; mobile phase: gradient elution of $10 \sim 100 \% \mathrm{CH}_{3} \mathrm{CN}$ in $0.1 \%$ TFA in 20 minutes; flow rate: $4 \mathrm{ml} /$ minute) to afford $2.0 \mathrm{mg}$ of grixazone B (2).

2: HR-ESIMS $m / z \quad 418.0701 \quad(\mathrm{M}+\mathrm{H})^{+}$(Calcd for $\mathrm{C}_{18} \mathrm{H}_{16} \mathrm{~N}_{3} \mathrm{O}_{7} \mathrm{~S}, 418.0709$ ); ${ }^{1} \mathrm{H}$ and ${ }^{13} \mathrm{C}$ NMR (Table 1).

\section{Results and Discussion}

Induction of Yellow Pigment Production

by Phosphate Depletion

We have observed that $S$. griseus produces a diffusible yellow pigment when cultured on a phosphate-depleted SMM agar plate ${ }^{4)}$. No yellow pigment production was observed on a standard SMM agar plate containing $2.5 \mathrm{mM}$ $\mathrm{KH}_{2} \mathrm{PO}_{4}$. To clarify the relationship between the yellow pigment production and phosphate concentration in the medium, we analyzed production of the pigment by $S$. griseus cultivated in minimal liquid medium containing different concentrations of phosphate. As shown in Fig. 1A, a clear correlation between the growth of $S$. griseus and the phosphate concentration was observed. In the presence of $2.5 \mathrm{mM} \mathrm{KH}_{2} \mathrm{PO}_{4}$, the strain grew most vigorously. As the concentration of $\mathrm{KH}_{2} \mathrm{PO}_{4}$ decreased, the growth rate became low. In the absence of $\mathrm{KH}_{2} \mathrm{PO}_{4}$, the strain could not grow (data not shown). Production of the yellow pigment was examined by measuring the absorbance at $433 \mathrm{~nm}$ $\left(\mathrm{A}_{433}\right)$ of the culture supernatant (Fig. 1B). In the presence of $0.1,0.25$, and $0.5 \mathrm{~mm} \mathrm{KH}_{2} \mathrm{PO}_{4}$, yellow pigment production began on day 2 (early stationary phase), while it began on day 3 in the presence of $1.0 \mathrm{~mm} \mathrm{KH}_{2} \mathrm{PO}_{4}$. This may be because the concentration of phosphate at day 2 in the medium containing $1.0 \mathrm{~mm} \mathrm{KH}_{2} \mathrm{PO}_{4}$ dose not decrease to the critical level that induces the pigment production. In the presence of $2.5 \mathrm{mM} \mathrm{KH}_{2} \mathrm{PO}_{4}$, no yellow pigment 
Fig. 1. Growth (A) and production of yellow pigment (B) of $S$. griseus IFO13350 cultivated in SMM containing different concentrations of $\mathrm{KH}_{2} \mathrm{PO}_{4}$.

\section{A}

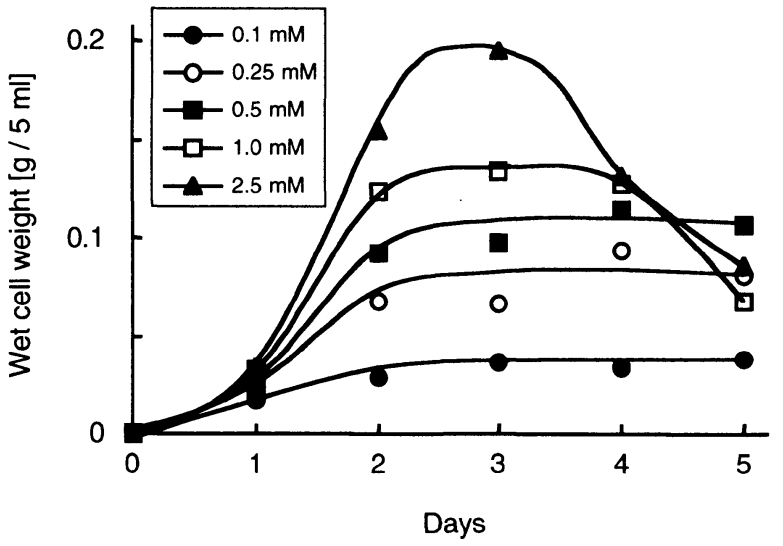

B

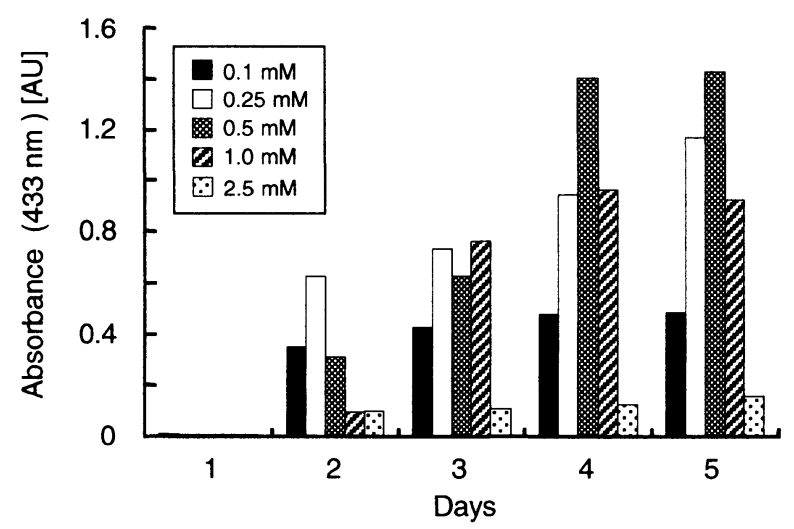

production was observed even at day 5. Although a small increase of $\mathrm{A}_{433}$ was observed in the culture supernatant, the yellow pigment was not detected by HPLC analysis (data not shown). These results indicated that the yellow pigment production was induced by phosphate depletion.

\section{HPLC Analysis of the Yellow Pigment}

For isolation and structure determination of the yellow pigment, the culture size was increased from $100 \mathrm{ml}$ to 1 liter and the effect of phosphate on the pigment production was examined again. In the scale-up culture, the yellow pigment was not produced in the presence of $1.0 \mathrm{~mm}$ $\mathrm{KH}_{2} \mathrm{PO}_{4}$ even at day 5 . The pigment was produced when
Fig. 2. HPLC analysis of the supernatant obtained from the $S$. griseus culture grown for 5 days (A) and $U V$-visible spectra of grixazone $A$ and $B$ on a photodiode array detector (B).

A


B

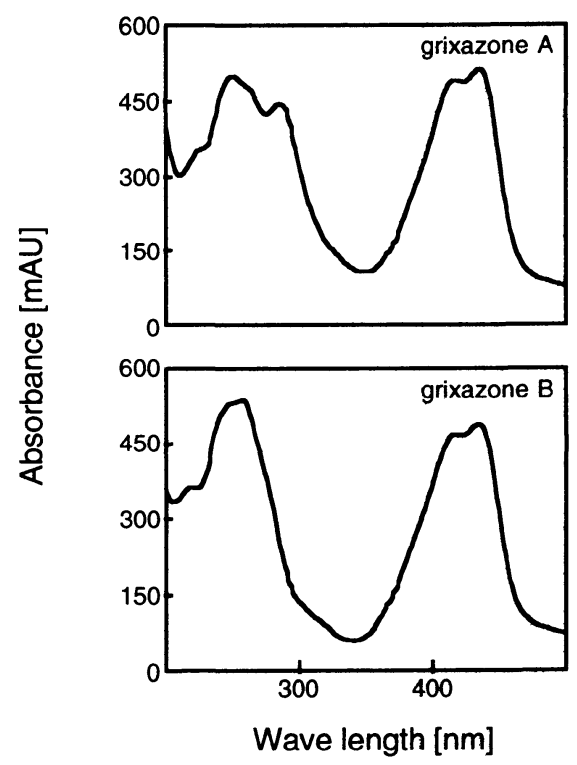

the concentration of $\mathrm{KH}_{2} \mathrm{PO}_{4}$ was below $0.75 \mathrm{~mm}$. HPLC analysis of the culture supernatant revealed that the pigment consisted of two major compounds, later 
designated as grixazone A and B (Fig. 2A). Similar UVvisible spectra of both compounds (Fig. 2B) suggested that they had a common chromophore. Interestingly, the ratio of the amounts of both compounds was varied depending on the phosphate concentration. Grixazone A was a major product in the presence of $0.25 \mathrm{mM} \mathrm{KH}_{2} \mathrm{PO}_{4}$, whereas grixazone $\mathrm{B}$ was a major product in the presence of $0.75 \mathrm{mM} \mathrm{KH}_{2} \mathrm{PO}_{4}$. In the presence of $0.5 \mathrm{mM} \mathrm{KH}_{2} \mathrm{PO}_{4}$, both compounds were produced in almost the same amount. The molecular mechanism for this observation remained to be elucidated. No yellow pigment production by an A-factordeficient strain (HH1) and an adpA-disrupted strain was confirmed by HPLC analysis of their culture supernatants (data not shown).

\section{Structure Determination of Grixazone A and B}

The molecular formula of grixazone A (1) was determined as $\mathrm{C}_{13} \mathrm{H}_{15} \mathrm{~N}_{3} \mathrm{O}_{6} \mathrm{~S}$ by HR-ESIMS and ${ }^{13} \mathrm{C}$ NMR spectra of $\mathbf{1}$. Analysis of NMR spectra of 1 revealed the presence of an aldehyde group and an $\mathrm{N}$-acetylamino acid residue (partial structure A, Fig. 3) in 1. Atomic composition and NMR data of the remaining part showed that the chromophore moiety of $\mathbf{1}$ showing a yellow color was a 2-aminophenoxazin-3-one residue, which was confirmed by comparison of its NMR data with those of 2aminophenoxazin-3-one ${ }^{10)}$. The $\delta$ values and coupling patterns of three protons on the benzene ring of the

Fig. 3. Partial structures (A) and (B), and HMBC correlation.

A

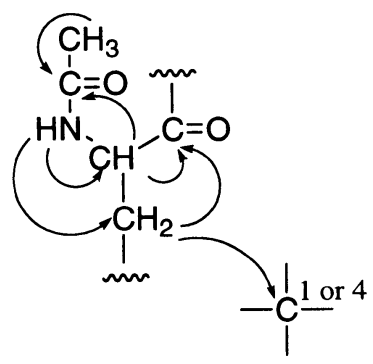

B

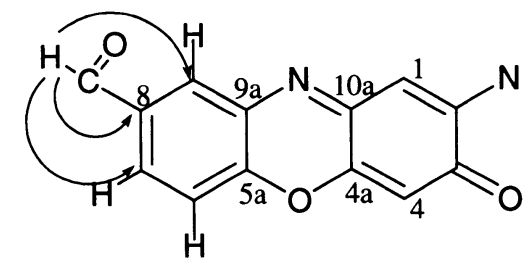

chromophore (Table 1) and the long-range couplings between the aldehyde proton and the ring carbons indicated that the aldehyde group is present at $\mathrm{C}-8$ of the chromophore $^{11)}$ (partial structure (B), Fig. 3). These results showed that 1 should have a carboxy group in the partial structure (A) and an amino group at C-2 of the partial structure (B) to fulfill the molecular formula of $\mathbf{1}$, and that the amino acid residue should connect to $\mathrm{C}-1$ or $\mathrm{C}-4$ of the partial structure (B) through a remaining sulfur atom. This connection was supported by a long-range coupling between the methylene protons of the $N$-acetylcysteine residue and the carbon with the $\delta$ value of 99.3 , but it was difficult to determine whether the carbon is $\mathrm{C}-1$ or $\mathrm{C}-4$ due to the similarities of $\delta_{\mathrm{H}}$ and $\delta_{\mathrm{C}}$ values at C- 1 and C-4 of the 2-aminophenoxazin-3-one moiety ${ }^{11)}$. The ${ }^{3} J_{\mathrm{C}, \mathrm{H}}$ and ${ }^{2} J_{\mathrm{C}, \mathrm{H}}$ couplings observed on the singlet proton $(\delta 6.47, \mathrm{H}-1$ or $\mathrm{H}-$ 4) were not useful to determine the position, and the ${ }^{4} J_{\mathrm{C}, \mathrm{H}}$ coupling between the proton and C-5a or C-9a was not observed. However, information of ${ }^{3} J_{\mathrm{H}, \mathrm{N}}$ coupling strongly suggested the connection at $\mathrm{C}-1$. Namely, a ${ }^{3} J_{\mathrm{H}, \mathrm{N}}$ coupling between $\mathrm{H}-9$ and $\mathrm{N}-10\left(\delta_{\mathrm{N}} 302.1, \mathrm{NH}_{3} \delta_{\mathrm{N}} 0.0\right)$ was observed in the ${ }^{1} \mathrm{H}_{-}{ }^{15} \mathrm{~N}$ HMBC spectrum of 1 , but that between the proton $(\delta 6.47)$ and $\mathrm{N}-10$ was not observed under the same measurement conditions. Thus, the structure of grixazone A (1) was determined as shown in Fig. 4, which is a novel phenoxazinone derivative.

Grixazone B (2) had a molecular formula of $\mathrm{C}_{13} \mathrm{H}_{15} \mathrm{~N}_{3} \mathrm{O}_{7} \mathrm{~S}$, which was larger than that of $\mathbf{1}$ by one oxygen atom. ${ }^{1} \mathrm{H}$ and ${ }^{13} \mathrm{C}$ NMR spectra of 2 were very similar to those of $\mathbf{1}$, but small differences between them were observed in signals around the benzene ring moiety. An aldehyde proton signal was not observed in ${ }^{1} \mathrm{H}$ NMR of 2 and a carbonyl carbon signal ( $\delta$ 166.3) was newly observed in the ${ }^{13} \mathrm{C}$ NMR spectrum of 2 instead of the signal at $\delta 191.8$ in the spectrum of $\mathbf{1}$. These data showed

Fig. 4. Structures of grixazone A (1) and B (2).<smiles>[R]c1ccc2oc3cc(=O)c(N)c(SCC(NC(C)=O)C(=O)O)c-3nc2c1</smiles>

grixazone $\mathrm{A}(\mathbf{1}): \mathrm{R}=\mathrm{CHO}$ grixazone $B(2): R=\mathrm{COOH}$ 
that 2 had the 8-carboxylic acid structure as shown in Fig. 4. This structure has been assigned to a compound with parasiticidal activity produced by Streptomyces sp. DSM 3813 in a patent ${ }^{12)}$. But details of structural determination of the compound have not been published. Assignments of protons and carbons in the NMR spectra of $\mathbf{1}$ and $\mathbf{2}$ are summarized in Table 1.

Antimicrobial Activity of Grixazone A and B

Antimicrobial activity of grixazone A and B was examined by using Saccharomyces cerevisiae TP-F0176, Candida albicans TP-F0594, Bacillus subtilis ATCC6633, Escherichia coli NIHJ JC-2, and Staphylococcus aureus 209P JC-1 as indicators. All strains grew normally on the standard media containing $100 \mu \mathrm{g} / \mathrm{ml}$ of grixazone $\mathrm{A}$ and $\mathrm{B}$, indicating that grixazone $\mathrm{A}$ and $\mathrm{B}$ show no apparent antimicrobial activity.

\section{Acknowledgements}

This work was supported by a grant from the Industrial Technology Research Grant Program in 2003 of the New Energy and Industrial Technology Development Organization of Japan (03A07002). We thank Dr. K. KushidA of Varian Co. Ltd. for measurement of the ${ }^{1} \mathrm{H}^{-15} \mathrm{~N}$ HMBC spectrum with Coldprobe and Dr. H. OnAKA and Dr. T. Furumal for providing us with the strains for the antimicrobial activity assays.

\section{References}

1) HoRINOUCHI, S.: $\gamma$-Butyrolactones that control secondary metabolism and cell differentiation in Streptomyces. In Cell-cell signaling in bacteria. Ed., Dunny, G. M. \& Winans, S. C., pp. 193 207. American Society for Microbiology, Washington DC, 1999

2) Horinouchi, S.: A microbial hormone, A-factor, as a master switch for morphological differentiation and secondary metabolism in Streptomyces griseus. Front. Biosci. 7: d2045 2057, 2002
3) Ohnishi, Y.; S. Kameyama, H. Onaka \& S. Horinouchi: The A-factor regulatory cascade leading to streptomycin biosynthesis in Streptomyces griseus: identification of a target gene of the A-factor receptor. Mol. Microbiol. 34: 102 111, 1999

4) Yamazaki, H.; Y. Ohnishi \& S. Horinouchi: An Afactor-dependent extracytoplasmic function sigma factor $\left(\sigma^{\mathrm{Ads} A}\right)$ that is essential for morphological development in Streptomyces griseus. J. Bacteriol. 182: 4596 4605, 2000

5) Horinouchi, S.; H. Onaka, H. Yamazaki, S. Kameyama \& Y. OHNISHI: Isolation of DNA fragments bound by transcriptional factors, AdpA and ArpA, in the A-factor regulatory cascade. Actinomycetologica 14: $37 \sim 42$, 2000

6) Kato, J.; A. Suzuki, H. Yamazaki, Y. OHnishi \& S. HORINOUCHI: Control by A-factor of a metalloendopeptidase gene involved in aerial mycelium formation in Streptomyces griseus. J. Bacteriol. 184: 6016 6025, 2002

7) YAMAZAKI, H.; Y. OHNISHI \& S. HORINOUCHI: Transcriptional switch-on by A-factor of $\operatorname{sig} A$ that is essential for spore-septum formation in Streptomyces griseus. J. Bacteriol. 185: 1273 1283, 2003

8) Yamazaki, H.; Y. Takano, Y. Ohnishi \& S. Horinouchi: $a m f R$, an essential gene for aerial mycelium formation, is a member of the AdpA regulon in the A-factor regulatory cascade in Streptomyces griseus. Mol. Microbiol. 50: 1173 1187, 2003

9) Hopwood, D. A.; M. J. BibB, K. F. Chater, C. J. Bruton, H. M. Kieser, D. J. Lydiate, C. P. Smith, J. M. Ward \& H. SChrempF: Genetic manipulation of Streptomyces: A laboratory manual. The John Innes Institute, Norwich, 1985

10) Hughes, M. A.; M. J. Baggs, J. Al-Dulayymi, M. S. BAIRD \& P. A. Williams: Accumulation of 2-aminophenoxazin-3-one-7-carboxylate during growth of Pseudomonas putida TW3 on 4-nitro-substituted substrates requires 4-hydroxylaminobenzoate lyase (PnbB). Appl. Environ. Microbiol. 68: 4965 4970, 2002

11) Gerber, N. N. \& H. L. Yale: Structure and syntheses of texazone, 2-( $N$-methylamino)-3H-phenoxazin-3-one8-carboxylic acid, an actinomycete metabolite. J. Antibiotics 36: 688 694, 1983

12) Axel, Z.; B.-M. Sabine, G. Susanne, V. Hartmut \& S. GERHARD: Preparation of phenoxazinone derivatives by fermentation with Streptomyces DSM 3813 and their use as parasiticides. Eur. Pat. Appl. EP260486, 1988 